Pacific

Journal of

Mathematics

\title{
A COHOMOLOGICAL INJECTIVITY RESULT FOR THE RESIDUAL AUTOMORPHIC SPECTRUM OF GL $_{n}$
}

\author{
HARALD GROBNER
}




\title{
A COHOMOLOGICAL INJECTIVITY RESULT FOR THE RESIDUAL AUTOMORPHIC SPECTRUM OF GL n $_{n}$
}

\author{
HARALD GROBNER
}

Let $\Pi$ be a cohomological residual automorphic representation of $\mathrm{GL}_{n} / F$, for $F$ an arbitrary number field. Let $q_{\min }$ be the lowest degree in which $\Pi$ has nonvanishing cohomology. We prove that the cohomology of $\Pi$ always injects into the cohomology of the corresponding locally symmetric space in degree $q_{\min }$. This extends the well-known result of Borel for cuspidal automorphic representations to all square-integrable automorphic representations in this certain degree. Moreover, we thereby improve a result of Rohlfs and Speh and confirm an idea of Harder.

\section{Introduction}

Let $F$ be any number field and let $G=\mathrm{GL}_{n} / F$. As it is well-known, the space of square-integrable automorphic forms of $G(\mathbb{A})$ decomposes into the space $\mathscr{A}_{\text {cusp }}(G)$ of cuspidal automorphic forms, and a natural complement, the space $\mathscr{A}_{\text {res }}(G)$ of residual automorphic forms. The latter are given by square-integrable residues of Eisenstein series and described in terms of representation theory by [Mœglin and Waldspurger 1989]. Let $\Pi$ be a residual automorphic representation of $G(\mathbb{A})$. We say that $\Pi$ is cohomological, if the ring of relative Lie algebra cohomology of $\Pi$ is nonvanishing with respect to some irreducible, finite-dimensional algebraic representation $M$ of $G$. See also Section 1C. Assume now that $\Pi$ is cohomological and let $q_{\min }$ be the lowest degree in which $\Pi$ has nonvanishing cohomology.

In this paper we prove that, in degree $q_{\min }$, the cohomology of $\Pi$ always injects into the cohomology of the locally symmetric space attached to $G$. This extends the well-known result of Borel [1981] for cuspidal automorphic representations to all square-integrable automorphic representations in this certain degree. The precise result reads as follows (see Theorem 4.1, also for unexplained notation):

Theorem. Let $G=\mathrm{GL}_{n} / F$ and let $\mathcal{M}$ be an irreducible, finite-dimensional, algebraic representation of $G$ on a complex vector space. Let $\{P\}$ be an associate class of proper parabolic $F$-subgroups of $G$ and let $\varphi_{P}$ be an associate class of

Grobner is supported by the Austrian Science Fund, project number P 25974-N25.

MSC2010: primary 11F70, 11F75, 22E47; secondary 11F67.

Keywords: automorphic cohomology, residual representation, general linear group. 
cuspidal automorphic representations of $L_{P}(\mathbb{A})$. Let $\Pi \hookrightarrow \mathscr{A}_{\mathrm{res}, \mathscr{g}}(G)$ be a residual automorphic representation of $G(\mathbb{A})$ with cuspidal support $\pi \in \varphi_{P}$, spanned by iterated residues of Eisenstein series at a point $v \in \check{\mathfrak{a}}_{P, \mathbb{C}}^{G}$ for which $v+\chi_{\tilde{\pi}}$ is annihilated by $\mathscr{g}$. The map in cohomology

$$
H^{q_{\min }}\left(\mathfrak{m}_{G}, K, \Pi \otimes \mathcal{M}\right) \longrightarrow H^{q_{\min }}\left(\mathfrak{m}_{G}, K, \mathscr{A}_{\mathscr{g},\{P\}, \varphi_{P}}(G) \otimes M\right),
$$

induced from the natural inclusion $\Pi \hookrightarrow \mathscr{A}_{\mathscr{F},\{P\}, \varphi_{P}}(G)$, is injective. In other words, the $\left(\mathfrak{m}_{G}, K\right)$-cohomology of a residual automorphic representation of $\mathrm{GL}_{n}(\mathbb{A})$ always embeds into $H^{\bullet}\left(G(F) \backslash G(\mathbb{A}) / A_{G}^{\mathbb{R}} K, \widetilde{M}\right)$ in its lowest, nonvanishing degree.

This improves a result of Rohlfs and Speh [2011] (see also our Remark 4.2) and confirms an idea of Harder. Moreover, it may be viewed as a refinement of one of our own results in [Grobner 2013]. Although we believe that it is interesting in its own right, we hope that it will also be of use in a forthcoming work of Harder and Raghuram on special values of Ranking-Selberg $L$-functions.

\section{Notation}

1A. Number fields and adeles. Let $F$ denote an arbitrary number field with set of places $S$. We write $S_{\infty}=S_{\mathbb{R}} \cup S_{\mathbb{C}}$ for the subset of archimedean places, where $S_{\mathbb{R}}$ denotes the set of real archimedean places and $S_{\mathbb{C}}$ denotes the set of complex archimedean places of $F$. We use $F_{v}$ for the topological completion of $F$ at $v \in S$. As usual, A stands for its ring of adeles.

1B. Algebraic groups. In this paper, $G:=\mathrm{GL}_{n} / F$ denotes the general linear group over $F$. We fix the usual Borel subgroup $B$ of upper triangular matrices with Levi decomposition $B=T U$. This choice defines the standard parabolic $F$-subgroups $P$ with Levi decomposition $P=L_{P} N_{P}$, where $L_{P} \supseteq T$ and $N_{P} \subseteq U$. Clearly, $L_{P} \cong \mathrm{GL}_{k_{1}} \times \cdots \times \mathrm{GL}_{k_{\ell}}$, with $\sum_{i=1}^{\ell} k_{i}=n$. We let $A_{P}=Z_{L_{P}}$ be the maximal $F$ split torus of $L_{P}$, satisfying $A_{P} \subseteq T$ and denote by $\mathfrak{a}_{P}$ (resp., $\mathfrak{a}_{P, \mathbb{C}}$ ) its Lie algebra (resp., its complexification $\mathfrak{a}_{P, \mathbb{C}}=\mathfrak{a}_{P} \otimes \mathbb{C}$ ). The respective duals are denotes $\check{\mathfrak{a}}_{P}$ and $\check{\mathfrak{a}}_{P, \mathbb{C}}$. The inclusion $A_{P} \subseteq T$ (resp., the restriction to $P$ ) defines $\mathfrak{a}_{P} \rightarrow \mathfrak{t}$ (resp., $\check{\mathfrak{a}}_{P} \rightarrow \check{\mathfrak{t}}$ ), which leads to direct sum decompositions $\mathfrak{t}=\mathfrak{a}_{P} \oplus \mathfrak{a}^{P}$ and $\check{\mathfrak{t}}=\check{\mathfrak{a}}_{P} \oplus \check{\mathfrak{a}}^{P}$. We let $\mathfrak{a}_{P}^{Q}:=\mathfrak{a}_{P} \cap \mathfrak{a}^{Q}$ and $\check{\mathfrak{a}}_{P}^{Q}:=\check{\mathfrak{a}}_{P} \cap \check{\mathfrak{a}}^{Q}$ for parabolic $F$-subgroups $Q$ and $P$. We write $H_{P}: L_{P}(\mathbb{A}) \rightarrow \mathfrak{a}_{P, \mathbb{C}}$ for the standard Harish-Chandra height function [Franke 1998, p. 185]. The group $L_{P}(\mathbb{A})^{1}:=\operatorname{ker} H_{P}$, admits a direct complement $A_{P}^{\mathbb{R}} \cong \mathbb{R}_{+}^{\operatorname{dim}_{P}}=\mathbb{R}_{+}^{\ell}$ in $L_{P}(\mathbb{A})$ whose Lie algebra is isomorphic to $\mathfrak{a}_{P} \cong \mathbb{R}^{\ell}$. With respect to a maximal compact subgroup $K_{\mathbb{A}} \subseteq G(\mathbb{A})$ in good position (see [Mœglin and Waldspurger 1995, I.1.4]), we obtain an extension $H_{P}: G(\mathbb{A}) \rightarrow \mathfrak{a}_{P, \mathbb{C}}$ to all of $G(\mathbb{A})$. 
1C. Lie groups and Lie algebras. The Lie algebra of a real Lie group is denoted by the same letter in gothic lowercase; thus $\mathfrak{g}_{\infty}=\mathfrak{g l}_{n}(\mathbb{R})^{\left|S_{\mathbb{R}}\right|} \oplus \mathfrak{g l}_{n}(\mathbb{C})^{\left|S_{\mathbb{C}}\right|}$ is the real Lie algebra of $G_{\infty}:=R_{F / \mathbb{Q}}(G)(\mathbb{R})$, and so on. We set $\mathfrak{m}_{G}:=\mathfrak{g}_{\infty} / \mathfrak{a}_{G}=$ $\operatorname{Lie}\left(G(\mathbb{A})^{1} \cap G_{\infty}\right)$ and denote by $\mathfrak{Z}\left(\mathfrak{g}_{\infty}\right)$ the center of the universal enveloping algebra $U\left(\mathfrak{g}_{\infty}\right)$ of $\mathfrak{g}_{\infty, \mathbb{C}}:=\mathfrak{g}_{\infty} \otimes_{\mathbb{R}} \mathbb{C}$. We will also use the notation $G_{v}$ for $G\left(F_{v}\right)$, $v \in S_{\infty}$, and similar for other local groups (such as $L_{P, v}$ etc.).

Let $K_{\infty} \subset G_{\infty}$ be a maximal compact subgroup (the archimedean factor of the maximal compact subgroup $K_{\mathbb{A}}$ of $G(\mathbb{A})$ in good position) and set once and for all $K:=K_{\infty}^{\circ}$, the connected component of the identity element. We refer the reader to [Borel and Wallach 1980, Chapter I] for the basic facts and notations concerning $\left(\mathfrak{m}_{G}, K\right)$-cohomology. If $H$ is any subgroup of $G_{\infty}$, we denote by $K_{H}$ the intersection $K \cap H$.

1D. Algebraic representations. In this paper, $M$ will always be a finite-dimensional irreducible algebraic representation of $G$ on a complex vector space. For simplicity, we will assume that $A_{G}^{\mathbb{R}}$ (and so $\mathfrak{a}_{G}$ ) acts trivially on $\mathcal{M}$. There is hence no difference between the $\left(\mathfrak{g}_{\infty}, K\right)$-module and the $\left(\mathfrak{m}_{G}, K\right)$-module defined by $\mathcal{M}$.

\section{Automorphic representations}

2A. Automorphic forms. Our notion of an automorphic form $f: G(\mathbb{A}) \rightarrow \mathbb{C}$ and of an automorphic representation of $G(\mathbb{A})$ is the one from [Borel and Jacquet 1979, 4.2 and 4.6]. Let $\mathscr{A}(G)$ be the space of all automorphic forms $f: G(\mathbb{A}) \rightarrow \mathbb{C}$ that are constant on the real Lie subgroup $A_{G}^{\mathbb{R}}$. By its very definition, every automorphic form is annihilated by some power of an ideal $\mathscr{S} \triangleleft \mathfrak{Z}\left(\mathfrak{g}_{\infty}\right)$ of finite codimension. We fix such an ideal $\mathscr{F}$ once and for all; as we will only be interested in cohomological automorphic forms, we take $\mathscr{g}$ to be the ideal which annihilates the contragredient representation $\mathcal{M}^{\vee}$ of $\mathcal{M}$ (see Section 1C) and denote by

$$
\mathscr{A}_{\mathscr{g}}(G) \subset \mathscr{A}(G)
$$

the space consisting of those automorphic forms that are annihilated by some power of $\mathscr{g}$. Clearly, $\mathscr{A}_{\mathscr{g}}(G)$ carries commuting $\left(\mathfrak{g}_{\infty}, K\right)$ - and $G\left(\mathbb{A}_{f}\right)$-actions and hence defines an $\left(\mathfrak{m}_{G}, K, G\left(\mathbb{A}_{f}\right)\right)$-module. As such a module, any irreducible subquotient (that is, any automorphic representation) $\Pi$ decomposes as $\Pi \cong \Pi_{\infty} \otimes \Pi_{f}$.

2B. $L^{\mathbf{2}}$-automorphic forms. The $\left(\mathfrak{m}_{G}, K, G\left(\mathbb{A}_{f}\right)\right)$-submodule of all square-integrable automorphic forms in $\mathscr{A}_{\mathscr{F}}(G)$ is denoted $\mathscr{A}_{\mathrm{dis}, \mathscr{g}}(G)$. An irreducible subrepresentation of $\mathscr{A}_{\mathrm{dis}, \mathscr{F}}(G)$ will be called an $L^{2}$-automorphic representation (see [Borel 2007, 9.6]). If $\omega: Z_{G}(F) \backslash Z_{G}(\mathbb{A}) \rightarrow \mathbb{C}^{*}$ is a continuous character of the center $Z_{G}$ of $G$, we let $\mathscr{A}_{\text {dis, } \mathscr{G}}(G, \omega)$ be the space of square-integrable automorphic forms with central character $\omega$. 
We further recall that $\mathscr{A}_{\text {dis, },}(G, \omega)$ decomposes as a direct sum of automorphic representations $\Pi$

$$
\mathscr{A}_{\mathrm{dis}, \mathscr{q}}(G, \omega) \cong \bigoplus \Pi
$$

which can be described as follows: According to [Mœglin and Waldspurger 1989], every summand $\Pi$ in the above decomposition is of the form $\Pi \cong J(P, \pi, v)$, where the latter stands for the (smooth, $K$-finite vectors in the) unique irreducible quotient of the (normalized) induced representation $I_{P(A)}^{G(A)}[\pi \otimes v]$, with inducing data $\pi$, a cuspidal automorphic representation of $L_{P}(\mathbb{A})$, and $v \in \check{\mathfrak{a}}_{P, \mathbb{C}}$. In fact, as is well-known, by [Mœglin and Waldspurger 1989, Théorème, p. 606], more can be said:

Theorem 2.1. Any $L^{2}$-automorphic representation of $G(\mathbb{A})$ is given by a triple $\left(L_{P}, \sigma, v\right)$, where

(1) $L_{P} \cong \mathrm{GL}_{k} \times \cdots \times \mathrm{GL}_{k}$, with $\ell k=n$;

(2) $\pi \cong \sigma \otimes \cdots \otimes \sigma$, with $\sigma$ a cuspidal automorphic representation of $\operatorname{GL}_{k}(\mathbb{A})$;

(3) $v=((\ell-1) / 2, \ldots,(1-\ell) / 2)$ in the coordinates given by the absolute value of the determinant of $\mathrm{GL}_{k}(\mathbb{A})$;

and no other triples determine an $L^{2}$-automorphic representation. The datum $\left(L_{P}, \sigma, v\right)$ is unique.

As a matter of fact, the space of $L^{2}$-automorphic forms decomposes as a direct sum

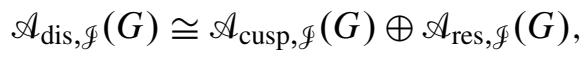

where $\mathscr{A}_{\text {cusp, } \mathscr{G}}(G)$ is the space of cuspidal automorphic forms in $\mathscr{A}_{\mathscr{G}}(G)$ and $\mathscr{A}_{\text {res, } \mathscr{F}}(G)$ denotes the space of residual automorphic forms in $\mathscr{A}_{\mathscr{g}}(G)$. More precisely, adding a central character $\omega$ to this datum, according to the theorem above, $\mathscr{A}_{\text {res, } \mathscr{~}}(G, \omega)$ is the direct sum of all $L^{2}$-automorphic representations given by a triple $\left(L_{P}, \sigma, \nu\right)$, with $P$ proper.

2C. Parabolic supports. Let $\{P\}$ be the associate class of the parabolic $F$-subgroup $P$. It consists by definition of all parabolic $F$-subgroups $Q=L_{Q} N_{Q}$ of $G$ for which $L_{Q}$ and $L_{P}$ are conjugate by an element in $G(F)$. We denote by $\mathscr{A}_{\mathscr{f},\{P\}}(G)$ the space of all $f \in \mathscr{A}_{\mathscr{f}}(G)$ that are negligible along every parabolic $F$-subgroup $Q \notin\{P\}$. (For the sake of completeness, we recall that the latter condition means that for all $g \in G(\mathbb{A})$, the function $L_{Q}(\mathbb{A}) \rightarrow \mathbb{C}$ given by $l \mapsto f_{Q}(l g)$ is orthogonal to the space of cuspidal functions on $L_{P}(F) A_{G}^{\mathbb{R}} \backslash L_{P}(\mathbb{A})$.) There is the following decomposition of $\mathscr{A}_{\mathscr{f}}(G)$ as an $\left(\mathfrak{m}_{G}, K, G\left(\mathbb{A}_{f}\right)\right.$ )-module (see [Borel et al. 1996, Theorem 2.4] or [Borel 2007, 10.3]), first established by Langlands:

$$
\mathscr{A}_{\mathscr{g}}(G) \cong \bigoplus_{\{P\}} \mathscr{A}_{\mathscr{F},\{P\}}(G)
$$


2D. Cuspidal supports. The various summands $\mathscr{A}_{\mathscr{G},\{P\}}(G)$ can be decomposed even further. To this end, recall from [Franke and Schwermer 1998, 1.2] the notion of an associate class $\varphi_{P}$ of cuspidal automorphic representations of the Levi subgroups of the elements in the class $\{P\}$. Therefore, let $\{P\}$ be represented by $P=L N$. Then the associate classes $\varphi_{P}$ may be parametrized by pairs of the form $(\Lambda, \tilde{\pi})$, where

(1) $\tilde{\pi}$ is a unitary cuspidal automorphic representation of $L(\mathbb{A})$, whose central character vanishes on the group $A_{P}^{\mathbb{R}}$;

(2) $\Lambda: A_{P}^{\mathbb{R}} \rightarrow \mathbb{C}^{*}$ is a Lie group character; and

(3) the infinitesimal character $\chi_{\tilde{\pi}}$ of $\tilde{\pi}_{\infty}$ and the derivative $d \Lambda \in \check{\mathfrak{a}}_{P, \mathbb{C}}$ of $\Lambda$ are compatible with the action of $\mathscr{S}$ (see [loc. cit.]).

Each associate class $\varphi_{P}$ may thus be represented by a cuspidal automorphic representation

$$
\pi:=\tilde{\pi} \otimes e^{\left\langle d \Lambda, H_{P}(\cdot)\right\rangle}
$$

of $L(\mathbb{A})$. Given $\varphi_{P}$, represented by a cuspidal representation $\pi$ of the above form, an $\left(\mathfrak{m}_{G}, K, G\left(\mathbb{A}_{f}\right)\right)$-submodule

$$
\mathscr{A}_{\mathscr{G},\{P\}, \varphi_{P}}(G)
$$

of $\mathscr{A}_{\mathscr{G},\{P\}}(G)$ was defined in Section 1.3 of [Franke and Schwermer 1998] as the span of all possible holomorphic values or residues of all Eisenstein series attached to $\tilde{\pi}$, evaluated at the point $\lambda=d \Lambda$, together with all their derivatives. This definition is independent of the choice of the representatives $P$ and $\pi$, thanks to the functional equations satisfied by the Eisenstein series considered. For details, we refer the reader to Sections 1.2-1.4 of the same paper.

The following refined decomposition as $\left(\mathfrak{m}_{G}, K, G\left(\mathbb{A}_{f}\right)\right)$-modules of the spaces $\mathscr{A}_{\mathscr{G},\{P\}}(G)$ of automorphic forms was obtained in [Franke and Schwermer 1998, Theorem 1.4]:

$$
\mathscr{A}_{\mathscr{F},\{P\}}(G) \cong \bigoplus_{\varphi_{P}} \mathscr{A}_{\mathscr{F},\{P\}, \varphi_{P}}(G) .
$$

2E. Quadruples in the refined version of Franke's filtration. A definition of the integer-valued function $T$ on the set of automorphic exponents is given in [Franke 1998, p. 233]. Because the technicalities are of little consequence to this paper, we won't repeat this definition here, but refer the reader to the original paper. The important fact is that we may assume a fixed choice of $T$ making the length $m=m(\{P\})$ of the corresponding filtration of $\mathscr{A}_{\mathscr{g},\{P\}}(G)$ minimal, as in our paper [Grobner 2013, 3.1 on p. 1072].

Given a cuspidal support $\varphi_{P}$, we will need the following collection of data, as was already introduced in [Grobner 2013, 3.2]. Let $M_{\mathscr{F},\{P\}, \varphi_{P}}$ be the set of 
quadruples $(R, \Pi, \nu, \lambda)$, with

(1) $R$ a standard parabolic $F$-subgroup of $G$ containing a representative of $\{P\}$;

(2) $\Pi$ a unitary discrete series automorphic representation of $L_{R}(\mathbb{A})$ with cuspidal support determined by $\varphi_{P}$, spanned by iterated residues of Eisenstein series at the point $v \in \check{\mathfrak{a}}_{P, \mathbb{C}}^{R}$; and

(3) $\lambda \in \check{\mathfrak{a}}_{R, \mathbb{C}}$ such that $\Re e(\lambda) \in \overline{\mathfrak{a}}_{R}^{G+}$, the closed positive Weyl chamber in $\check{\mathfrak{a}}_{R}^{G}$, and such that $\lambda+v+\chi_{\tilde{\pi}}$ is annihilated by $\mathscr{g}$.

We point out that with this definition, although not entirely obvious, one can show that $T$ is well-defined on $\Re e(\lambda)_{+}$; [Franke 1998, p. 233]. Therefore, taking this for granted, it makes sense to define

$$
M_{\mathscr{f},\{P\}, \varphi_{P}}^{(j)}:=\left\{(R, \Pi, \nu, \lambda) \mid T\left(\Re e(\lambda)_{+}\right)=j\right\} .
$$

These sets of quadruples $M_{\mathcal{F},\{P\}, \varphi_{P}}^{(j)}$ originate from [Franke 1998, pp. 218, 233-234]. There, however, only the parabolic support $\{P\}$ and not the cuspidal support $\varphi_{P}$ was taken into account.

\section{Automorphic cohomology}

3A. Cohomology of locally symmetric spaces. We let

$$
S:=G(F) A_{G}^{\mathbb{R}} \backslash G(\mathbb{A}) / K
$$

be the projective limit of the "locally symmetric spaces" attached to $G$. Starting from the algebraic representation $\mathcal{M}$, one obtains a sheaf $\widetilde{M}$ on $S$ by letting $\widetilde{M}$ be the sheaf with espace étalé $G(\mathbb{A}) / A_{G}^{\mathbb{R}} K \times_{G(F)} M$ with the discrete topology on $M$. We write $H^{q}(S, \widetilde{\mathcal{M}})$ for the corresponding space of sheaf cohomology (in degree $q$ ).

3B. Automorphic cohomology. We recall that the $G\left(\mathbb{A}_{f}\right)$-module

$$
H^{q}\left(\mathfrak{m}_{G}, K, \mathscr{A}_{\mathscr{g}}(G) \otimes \mathcal{M}\right)
$$

is called the automorphic cohomology of $G$ in degree $q$. From Sections $2 \mathrm{C}$ and $2 \mathrm{D}$ we know that it inherits a direct sum decomposition

$$
\begin{aligned}
H^{q}\left(\mathfrak{m}_{G}, K, \mathscr{A}_{\mathscr{f}}(G) \otimes M\right) & \cong \bigoplus_{\{P\}} H^{q}\left(\mathfrak{m}_{G}, K, \mathscr{A}_{\mathscr{F},\{P\}}(G) \otimes \mathcal{M}\right) \\
& \cong \bigoplus_{\{P\}} \bigoplus_{\varphi_{P}} H^{q}\left(\mathfrak{m}_{G}, K, \mathscr{A}_{\mathscr{F},\{P\}, \varphi_{P}}(G) \otimes M\right) .
\end{aligned}
$$

The summand $H^{q}\left(\mathfrak{m}_{G}, K, \mathscr{A}_{\mathscr{q},\{G\}}(G) \otimes \mathcal{M}\right)$ attached to $\{G\}$ consists precisely of all cuspidal automorphic forms in $\mathscr{A}_{\mathscr{g}}(G)$. 
Conjectured by Harder and Borel and proved by Franke [1998, Theorem 18], the following result which links automorphic cohomology with the sheaf cohomology of $S$ :

Theorem 3.1. There is an isomorphism of $G\left(\mathbb{A}_{f}\right)$-modules

$$
H^{q}(S, \widetilde{\mathcal{M}}) \cong H^{q}\left(\mathfrak{m}_{G}, K, \mathscr{A}_{\mathscr{g}}(G) \otimes \mathcal{M}\right) .
$$

The latter results brings us back to the more geometric point of view of cohomology, presented in Section 3A.

3C. Certain bounds in cohomology. Let $R=L_{R} N_{R}$ be a standard parabolic subgroup of $G$ and $v$ an archimedean place of $F$. We write

$$
\mathfrak{l}_{R, v} \cap \mathfrak{m}_{G}=\mathfrak{l}_{R, v}^{\mathrm{ss}} \oplus\left(\mathfrak{a}_{R, v} \cap \mathfrak{m}_{G}\right) \quad \text { and } \quad \mathfrak{k}_{L_{R}}^{\mathrm{ss}}:=\mathfrak{k}_{L_{R, v}} \cap \mathfrak{l}_{R, v}^{\mathrm{ss}} .
$$

Now, given an irreducible, admissible $L_{R, v}$-representation $\pi_{v}$, let $q\left(L_{R, v}, \pi_{v}\right)$ be the smallest degree in which $\pi_{v}$ has nontrivial $\left(\mathfrak{l}_{R, v}^{\mathfrak{s s}}, \mathfrak{k}_{L_{R, v}}^{\mathrm{ss}}\right)$-cohomology, twisted by an irreducible, finite-dimensional, algebraic representation of $L_{R, v}$. If there is no such coefficient module, then we let $q\left(L_{R, v}, \pi_{v}\right)=0$. (This number was denoted " $m\left(L_{R, v}, \pi_{v}\right)$ " in [Grobner 2013].) Similarly, we write $q\left(L_{R, \infty}, \pi_{\infty}\right):=$ $\sum_{v \in S_{\infty}} q\left(L_{R, v}, \pi_{v}\right)$.

Let $\{P\}$ be an associate class of proper parabolic $F$-subgroups of $G$, and $\varphi_{P}$ an associate class of cuspidal automorphic representations of $L_{P}(\mathbb{A})$. We define $q_{\text {res }}:=\min _{0 \leq j<m}\left(\min _{(R, \Pi, v, \lambda) \in M_{\mathcal{F},\{P\}, \varphi_{P}}^{(j)}}\left(\sum_{v \in S_{\infty}}\left\lceil\frac{1}{2} \operatorname{dim}_{\mathbb{R}} N_{R}\left(F_{v}\right)\right\rceil+q\left(L_{R, v}, \Pi_{v}\right)\right)\right)$.

Of course, although not reflected in the notation, $q_{\text {res }}$ depends on the support $\{P\}$ and $\varphi_{P}$. This rather complicatedly defined number (see [Grobner 2013, 6.1] for the original source) serves as a certain bound of degrees of cohomology, as we proved in the same paper. Indeed, boiled down to the case of $G=\mathrm{GL}_{n}$ here, in Corollary 17 of that paper, we showed the following result:

Theorem 3.2. Let $G=\mathrm{GL}_{n} / F$ and let $\mathcal{M}$ be an irreducible, finite-dimensional, algebraic representation of $G$ on a complex vector space. Let $\{P\}$ be an associate class of proper parabolic $F$-subgroups of $G$ and let $\varphi_{P}$ be an associate class of cuspidal automorphic representations of $L_{P}(\mathbb{A})$. Let $\Pi \hookrightarrow A_{\text {res, }, ~}(G)$ be a residual automorphic representation of $G(\mathbb{A})$ with cuspidal support $\pi \in \varphi_{P}$, spanned by iterated residues of Eisenstein series at a point $v \in \check{\mathfrak{a}}_{P, \mathbb{C}}^{G}$, for which $v+\chi_{\tilde{\pi}}$ is annihilated by F. Then, the map in cohomology

$$
H^{q}\left(\mathfrak{m}_{G}, K, \Pi \otimes \mathcal{M}\right) \longrightarrow H^{q}\left(\mathfrak{m}_{G}, K, \mathscr{A}_{\mathscr{g},\{P\}, \varphi_{P}}(G) \otimes M\right),
$$

induced from the natural inclusion $\Pi \hookrightarrow \mathscr{A}_{\mathscr{G},\{P\}, \varphi_{P}}(G)$, is injective in all degrees $0 \leq q<q_{\text {res }}=q_{\text {res }}\left(\{P\}, \varphi_{P}\right)$. 
The latter theorem will be the key result for the proof of our main result of this article in the next section.

\section{The main result}

4A. Let $\Pi \hookrightarrow \mathscr{A}_{\text {res }, \mathscr{q}}(G)$ be a residual automorphic representation of $G(\mathbb{A})$. Recall from Section $3 C$ our notation $q\left(G_{\infty}, \Pi_{\infty}\right)$ for the minimal degree in which $\Pi_{\infty}$ has nontrivial $\left(\mathfrak{m}_{G}, K\right)$-cohomology with respect to an irreducible, finite-dimensional, algebraic representation of $G$. For sake of simplicity, since the group $G$ and the representation $\Pi$ are clear from the context, we will write $q_{\min }:=q\left(G_{\infty}, \Pi_{\infty}\right)$ for this minimal degree.

Theorem 4.1. Let $G=\mathrm{GL}_{n} / F$ and let $\mathcal{M}$ be an irreducible, finite-dimensional, algebraic representation of $G$ on a complex vector space. Let $\{P\}$ be an associate class of proper parabolic $F$-subgroups of $G$ and let $\varphi_{P}$ be an associate class of cuspidal automorphic representations of $L_{P}(\mathbb{A})$. Let $\Pi \hookrightarrow \mathscr{A}_{\mathrm{res}, \Phi}(G)$ be a residual automorphic representation of $G(\mathbb{A})$ with cuspidal support $\pi \in \varphi_{P}$, spanned by iterated residues of Eisenstein series at a point $v \in \check{\mathfrak{a}}_{P, \mathbb{C}}^{G}$, for which $v+\chi_{\tilde{\pi}}$ is annihilated by $\mathscr{F}$. The map in cohomology

$$
H^{q_{\min }}\left(\mathfrak{m}_{G}, K, \Pi \otimes \mathcal{M}\right) \longrightarrow H^{q_{\min }}\left(\mathfrak{m}_{G}, K, \mathscr{A}_{\mathscr{F},\{P\}, \varphi_{P}}(G) \otimes M\right),
$$

induced from the natural inclusion $\Pi \hookrightarrow \mathscr{A}_{\mathscr{g},\{P\}, \varphi_{P}}(G)$, is injective. In other words, the $\left(\mathfrak{m}_{G}, K\right)$-cohomology of a residual automorphic representation of $\mathrm{GL}_{n}(\mathbb{A})$ always embeds into $H^{\bullet}(S, \widetilde{M})$ in its lowest, nonvanishing degree.

Remark 4.2. The reader should not confuse this theorem with [Rohlfs and Speh 2011, Theorem IV.4] and with [Grobner 2013, Theorem 22], where seemingly similar results were shown. In fact, Theorem 4.1 above is a improvement as well as a refinement of both of these theorems: First of all, here we show that the cohomology of a residual automorphic representation of $\mathrm{GL}_{n}(\mathbb{A})$ always injects into $H^{q}(S, \tilde{M})$ in its lowest nonvanishing degree and hence give a precise description of its nontrivial contribution. (Moreover, in contrast to [Rohlfs and Speh 2011], we allow any number field $F$ and any coefficient module $M$.) Secondly, we also obtain an improvement of the bound of degrees of cohomology given in [Grobner 2013, Theorem 22].

The proof of this theorem consists of two steps. First, we determine the minimal degree $q_{\min }$ explicitly for all cohomological residual automorphic representations of $G(\mathbb{A})$. Secondly, we make the effort and calculate our bound $q_{\text {res }}=q_{\text {res }}\left(\{P\}, \varphi_{P}\right)$ for given support $\{P\}, \varphi_{P}$ and show that it is always strictly greater than $q_{\min }$. The theorem is then a consequence from Theorem 3.2. As the reader will see, we will have to distinguish the case of a real archimedean place and a complex archimedean place. 


\section{Proof of main theorem: determination of $q_{\min }$}

5A. Let $\Pi \hookrightarrow \mathscr{A}_{\text {res, } \mathscr{G}}(G)$ be a residual automorphic representation of $G(\mathbb{A})$. By Theorem 2.1 it is given by a triple $\left(L_{P}, \pi, v\right)$, where $L_{P} \cong \mathrm{GL}_{k} \times \cdots \times \mathrm{GL}_{k}, \ell k=n$, and $\pi \cong \sigma \otimes \cdots \otimes \sigma$ is a cuspidal automorphic representation of $L_{P}(\mathbb{A})$. If $\Pi_{\infty}$ is cohomological with respect to $\mathcal{M}$, then $\pi_{\infty}$ is cohomological, too. As the only cohomological generic representations of $G_{\infty}$ are essentially tempered, we see that $\pi_{\infty}$ is essentially tempered. Hence, by its very construction, $\Pi_{\infty}$ is the Langlands quotient given by the triple $\left(L_{P, \infty}, \pi_{\infty}, v\right)$. Of course this also holds locally at $v \in S_{\infty}$.

Let now be $v \in S_{\mathbb{R}}$. Then $\Pi_{v}$ comes under the purview of the Vogan-Zuckerman classification of cohomological representations in terms of $A_{\mathfrak{q}}(\lambda)$-modules. We assume that the reader is familiar with this theory and refer to [Vogan and Zuckerman 1984] and [Knapp and Vogan 1995]. We write $\mathfrak{q}=\mathfrak{l} \oplus \mathfrak{u}$ for the Levi decomposition of the complex parabolic subalgebra $\mathfrak{q}$ of $\mathfrak{g}_{v, \mathbb{C}}$. By [Knapp and Vogan 1995, Chapter IV, Proposition 4.76], $\mathfrak{u}$ is the direct sum of certain root-eigenspaces, all of them one-dimensional. Hence, $\operatorname{dim}_{\mathbb{C}} \mathfrak{u}$ is the number of roots appearing in $\mathfrak{u}$. Moreover, from $\Pi_{v}$ being the Langlands quotient given by the triple $\left(L_{P}(\mathbb{R}), \pi_{v}, v\right)$, we derive that

$$
\mathfrak{l} \cong \begin{cases}\mathfrak{g l}_{\ell}(\mathbb{C})^{k / 2} & \text { for } k \text { even } \\ \mathfrak{g l}_{\ell}(\mathbb{C})^{(k-1) / 2} \oplus \mathfrak{g l}_{\ell}(\mathbb{R}) & \text { for } k \text { odd }\end{cases}
$$

see [Vogan and Zuckerman 1984, Theorem 6.16]. It is now an easy combinatorial exercise, using [Knapp and Vogan 1995, IV, Proposition 4.76] to show that the number of roots appearing in $\mathfrak{u}$ (and hence $\operatorname{dim}_{\mathbb{C}} \mathfrak{u}$ ) equals

$$
\operatorname{dim}_{\mathbb{C}} \mathfrak{U}= \begin{cases}\frac{1}{4} n(n-\ell+1) & \text { for } k \text { even, } \\ \frac{1}{4}(n(n-\ell+1)-\ell) & \text { for } k \text { odd. }\end{cases}
$$

Because in the case of $\mathfrak{g}=\mathfrak{g l}_{n}(\mathbb{R})$ all roots showing up in $\mathfrak{u}$ are noncompact, Theorem 5.5 of [Vogan and Zuckerman 1984] implies that the minimal degree in which $\Pi_{v} \cong A_{\mathfrak{q}}(\lambda)$ has nontrivial cohomology is precisely $\operatorname{dim}_{\mathbb{C}} \mathfrak{u}$.

Now let $v \in S_{\mathbb{C}}$. Then, by [Enright 1979], $\Pi_{v}$ is fully induced and so the minimal degree in which $\Pi_{v}$ has nontrivial cohomology is readily computed using [Borel and Wallach 1980, Chapter III, Theorem 3.3]. Summarizing, we have shown:

Proposition 5.1. Let $\Pi \hookrightarrow A_{\mathrm{res}, \mathscr{G}}(G)$ be a residual automorphic representation of $G(\mathbb{A})$ that is $\left(\mathfrak{m}_{G}, K\right)$-cohomological with respect to $\mathcal{M}$. Assume that $\Pi$ is given by the triple $\left(L_{P}, \pi, v\right)$, where $L_{P} \cong \mathrm{GL}_{k} \times \cdots \times \mathrm{GL}_{k}, \ell k=n$. Then

$$
q_{\text {min }}= \begin{cases}\left|S_{\mathbb{R}}\right| \cdot \frac{1}{4} n(n-\ell+1)+\left|S_{\mathbb{C}}\right| \cdot \frac{1}{2} n(n-\ell) & \text { for } k \text { even, } \\ \left|S_{\mathbb{R}}\right| \cdot \frac{1}{4}(n(n-\ell+1)-\ell)+\left|S_{\mathbb{C}}\right| \cdot \frac{1}{2} n(n-\ell) & \text { for } k \text { odd } .\end{cases}
$$




\section{Proof of main theorem: determination of $q_{\text {res }}$}

6A. Reduction to maximal parabolics. This step is much more technical in nature. We will have to make many case-by-case distinctions to actually calculate $q_{\text {res }}$. As a first result towards the determination of $q_{\text {res }}$, we shall need the following result:

Lemma 6.1. For every proper support $\{P\}, L_{P} \cong \mathrm{GL}_{k}^{\ell}, \varphi_{P}$ and every $j, 0 \leq j<m$, the minimum

$$
\min _{(R, \Pi, v, \lambda) \in M_{\mathcal{q},\{P\}, \varphi_{P}}^{(j)}}\left(\sum_{v \in S_{\infty}}\left\lceil\frac{1}{2} \operatorname{dim}_{\mathbb{R}} N_{R}\left(F_{v}\right)\right\rceil+q\left(L_{R, v}, \Pi_{v}\right)\right)
$$

is obtained at a maximal parabolic subgroup $R$.

Proof. We will prove this by checking that the number

$$
n\left(R_{v}\right):=\left\lceil\frac{1}{2} \operatorname{dim}_{\mathbb{R}} N_{R}\left(F_{v}\right)\right\rceil+q\left(L_{R, v}, \Pi_{v}\right)
$$

decreases for all $v \in S_{\infty}$ as we increase the parabolic subgroup $R$, that is, as we form the union of two diagonal blocks $a \cdot k$ and $b \cdot k$ to a block of size $(a+b) \cdot k$. We will write $R(a, b)$ for the first parabolic subgroup, that is, the one having two diagonal blocks of size $a \cdot k$ and $b \cdot k$, and $R(a+b)$ for the second parabolic subgroup, that is, the one having a diagonal block of size $(a+b) \cdot k$, containing the two diagonal blocks of size $a \cdot k$ and $b \cdot k$, instead.

Now, let $v \in S_{\mathbb{R}}$. We have to distinguish several cases. The first three, where both $a$ and $b$ are assumed to be greater than or equal to one, are checked using Proposition 5.1: As cohomology satisfies the Künneth rule, we computed the degree $q\left(L_{R, v}, \Pi_{v}\right)$ for a given quadruple $(R, \Pi, v, \lambda) \in M_{\mathscr{F},\{P\}, \varphi_{P}}^{(j)}$ in this proposition. The dimension of the unipotent radical is easily computed for each parabolic subgroups $R(a, b)$ and $R(a+b)$. Putting this together, we obtain:

Case 1: $k$ even, $a, b \geq 2$.

$$
\begin{aligned}
n\left(R(a, b)_{v}\right)-n\left(R(a+b)_{v}\right) & =\frac{1}{4} a k(a k-a+1)+\frac{1}{4} b k(b k-b+1)+\left\lceil\frac{1}{2} a b k^{2}\right\rceil \\
& =\frac{1}{2} a b k .
\end{aligned}
$$

Case 2: $k$ odd, $a, b \geq 2, a$ or $b$ even.

$$
n\left(R(a, b)_{v}\right)-n\left(R(a+b)_{v}\right)=\frac{1}{2} a b k .
$$

Case 3: $k$ odd, $a, b \geq 2, a$ and $b$ odd.

$$
n\left(R(a, b)_{v}\right)-n\left(R(a+b)_{v}\right)=\frac{1}{2}(a b k+1) .
$$

The remaining cases, namely when $b=1$, have a cuspidal automorphic component at the single $k$-block of $L_{R}$. This cuspidal automorphic representation has to 
be cohomological, whence its archimedean component at $v$ is tempered. The degree $q\left(L_{R, v}, \Pi_{v}\right)$ is now computed by Proposition 5.1 (for the residual representation of the block of size $a \cdot k$ ) and using [Borel and Wallach 1980, III, Proposition 5.3] (for the cuspidal representation of the block of size $k$ ), where the lowest degree of cohomology of tempered representations is determined. Finally, we obtain:

Case 4: $k$ even, $a \geq 2, b=1$.

$$
\begin{aligned}
n\left(R(a, b)_{v}\right)-n\left(R(a+b)_{v}\right)= & \frac{1}{4} a k(a k-a+1)+\frac{1}{2}\left(\frac{1}{2} k(k+1)-k+\left\lfloor\frac{1}{2} k\right\rfloor\right) \\
& \quad+\left\lceil\frac{1}{2} a k^{2}\right\rceil-\frac{1}{4}(a+1) k((a+1) k-(a+1)+1) \\
= & \frac{1}{2} a k .
\end{aligned}
$$

Case 5: $k$ odd, $a \geq 2$ even, $b=1$.

$$
n\left(R(a, b)_{v}\right)-n\left(R(a+b)_{v}\right)=\frac{1}{2} a k .
$$

Case 6: $k$ odd, $a \geq 2$ odd, $b=1$.

$$
n\left(R(a, b)_{v}\right)-n\left(R(a+b)_{v}\right)=\frac{1}{2}(a k+1) .
$$

Case 7: $k$ even, $a=b=1$.

$$
\begin{aligned}
n\left(R(a, b)_{v}\right)-n\left(R(a+b)_{v}\right) & =\left(\frac{1}{2} k(k+1)-k+\left\lfloor\frac{1}{2} k\right\rfloor+\left\lceil\frac{1}{2} a k^{2}\right\rceil\right)-\frac{1}{4} 2 k(2 k-1) \\
& =\frac{1}{2} k .
\end{aligned}
$$

Case 8: $k$ odd, $a=b=1$.

$$
n\left(R(a, b)_{v}\right)-n\left(R(a+b)_{v}\right)=\frac{1}{2}(k+1) .
$$

Summarizing all eight cases, we see that

$$
n\left(R(a, b)_{v}\right)-n\left(R(a+b)_{v}\right)>0 ;
$$

that is, $n\left(R_{v}\right)$ decreases, if $R$ increases.

Now, let $v \in S_{\mathbb{C}}$. This is the simple case, since $\Pi_{v}$ is fully induced and the cohomology of such representations is determined in [Borel and Wallach 1980, III, Theorem 3.3]. This is what we used in the proof of Proposition 5.1, where we also computed $q\left(L_{R, v}, \Pi_{v}\right)$ for a given quadruple $(R, \Pi, v, \lambda) \in M_{\mathscr{f},\{P\}, \varphi_{P}}^{(j)}$. Again, the dimension of the unipotent radical of the parabolic subgroups $R(a, b)$ and $R(a+b)$ is easily calculated. We obtain

$$
\begin{aligned}
n\left(R(a, b)_{v}\right)-n\left(R(a+b)_{v}\right)= & \frac{1}{2} a k(a k-a)+\frac{1}{2} b k(b k-b)+a b k^{2} \\
& -\frac{1}{2}(a+b) k((a+b) k-(a+b)) \\
= & a b k,
\end{aligned}
$$


now really for all cases of $a$ and $b$. Therefore, $n\left(R(a, b)_{v}\right)-n\left(R(a+b)_{v}\right)>0$, that is, $n\left(R_{v}\right)$ decreases, if $R$ increases also for $v \in S_{\mathbb{C}}$. This proves the lemma.

Proposition 6.3. For every proper support $\{P\}, L_{P} \cong \mathrm{GL}_{k}^{\ell}, \varphi_{P}$ and every $j$, $0 \leq j<m$, the minimum

$$
\min _{(R, \Pi, v, \lambda) \in M_{\mathcal{F},\{P\}, \varphi_{P}}^{(j)}}\left(\sum_{v \in S_{\infty}}\left\lceil\frac{1}{2} \operatorname{dim}_{\mathbb{R}} N_{R}\left(F_{v}\right)\right\rceil+q\left(L_{R, v}, \Pi_{v}\right)\right)
$$

occurs at the standard parabolic subgroup $R=L_{R} N_{R}$ with $L_{R} \cong \mathrm{GL}_{(\ell-1) k} \times \mathrm{GL}_{k}$. Proof. By Lemma 6.1, we only need to check that for $R=R((\ell-1) k, k)$, the number $n\left(R_{v}\right)$ is minimal among all maximal parabolic subgroups $R((\ell-a) k, a k)$ for all places $v \in S_{\infty}$. Precisely as in the proof of Lemma 6.1, this is again a lengthy exercise using Proposition 5.1 and [Borel and Wallach 1980, III, Proposition 5.3]. Their use is justified step-by-step, as in the proof of Lemma 6.1.

Let $v \in S_{\mathbb{R}}$. First of all, we check that

$$
n(R((\ell-1) k, k))= \begin{cases}\frac{1}{4}\left(n^{2}+(3-\ell) n-2 k\right) & \text { for } k \text { even, } \\ \frac{1}{4}\left(n^{2}+(3-\ell) n-2 k-\ell\right) & \text { for } k \text { odd }, \ell \text { odd }, \\ \frac{1}{4}\left(n^{2}+(3-\ell) n-2 k-\ell+2\right) & \text { for } k \text { odd, } \ell \text { even }\end{cases}
$$

Moreover, if $a \geq 2$, then $n(R((\ell-a) k, a k))$ is given by

$$
\frac{1}{4}\left(a^{2} k^{2}-a^{2} k+a k+(\ell-a)^{2} k^{2}-(\ell-a)^{2} k+(\ell-a) k+2 a(\ell-a) k^{2}\right)
$$

for $k$ even, by

$$
\frac{1}{4}\left(a^{2} k^{2}-a^{2} k+a k+(\ell-a)^{2} k^{2}-(\ell-a)^{2} k+(\ell-a) k-\ell+2 a(\ell-a) k^{2}\right)
$$

for $k$ odd and $a$ or $\ell-a$ even, and by

$\frac{1}{4}\left(a^{2} k^{2}-a^{2} k+a k+(\ell-a)^{2} k^{2}-(\ell-a)^{2} k+(\ell-a) k-\ell+2 a(\ell-a) k^{2}+2\right)$

for $k, a$ and $\ell-a$ odd.

The expression $n(R((\ell-a) k, a k))$ is a quadratic polynomial in $a$, with strictly negative leading coefficient. Hence, for $a \geq 2, n(R((\ell-a) k, a k))$ is minimal at $a=2$ (and $a=\ell-2$ ). We obtain

$$
n(R((\ell-2) k, 2 k))= \begin{cases}\frac{1}{4}\left(n^{2}+(5-\ell) n-8 k\right) & \text { for } k \text { even, } \\ \frac{1}{4}\left(n^{2}+(5-\ell) n-8 k-\ell\right) & \text { for } k \text { odd. }\end{cases}
$$

Comparing $n(R((\ell-2) k, 2 k))$ to $n(R((\ell-1) k, k))$, in the cases when either $k$ is even or $k$ is odd and $\ell$ is odd, we see that $n(R((\ell-2) k, 2 k)) \geq n(R((\ell-1) k, k))$ if and only if $\ell \geq 3$. But this is fine without loss of generality, since for $\ell=2$ the result holds trivially. If $k$ is odd and $\ell$ is even, $n(R((\ell-2) k, 2 k)) \geq n(R((\ell-1) k, k))$ if 
and only if $\ell \geq 4$. This is satisfied by the same reason, since $\ell \geq 3$ is assumed to be even, hence without loss of generality already $\ell \geq 4$.

Now, let $v \in S_{\mathbb{C}}$. Then

$$
n(R((\ell-a) k, a k))=\frac{n^{2}-(2 a-\ell) n-2 a^{2} k}{2},
$$

for all $a \geq 1$. Clearly, this is minimal at $a=1$ (and $a=\ell-1$ ).

Proposition 6.4. Let $\Pi \hookrightarrow \mathscr{A}_{\mathrm{res}, \mathscr{q}}(G)$ be a residual automorphic representation of $G(\mathbb{A})$, which is $\left(\mathfrak{m}_{G}, K\right)$-cohomological with respect to $M$. Assume that $\Pi$ is given by the triple $\left(L_{P}, \pi, v\right)$, where $L_{P} \cong \mathrm{GL}_{k} \times \cdots \times \mathrm{GL}_{k}, \ell k=n$. Then $q_{\text {res }}$ is given by

$$
\left|S_{\mathbb{R}}\right| \cdot \frac{1}{4}\left(n^{2}+(3-\ell) n-2 k\right)+\left|S_{\mathbb{C}}\right| \cdot \frac{1}{2}\left(n^{2}-(2-\ell) n-2 k\right)
$$

for $k$ even, by

$$
\left|S_{\mathbb{R}}\right| \cdot \frac{1}{4}\left(n^{2}+(3-\ell) n-2 k-\ell\right)+\left|S_{\mathbb{C}}\right| \cdot \frac{1}{2}\left(n^{2}-(2-\ell) n-2 k\right)
$$

for $k$ odd and $\ell$ odd, and by

$$
\left|S_{\mathbb{R}}\right| \cdot \frac{1}{4}\left(n^{2}+(3-\ell) n-2 k-\ell+2\right)+\left|S_{\mathbb{C}}\right| \cdot \frac{1}{2}\left(n^{2}-(2-\ell) n-2 k\right)
$$

for $k$ odd and $\ell$ even.

Proof. This holds by the definition of $q_{\text {res }}$ and Proposition 6.3.

6B. End of the proof of the Theorem 4.1. A direct comparison of $q_{\min }$ and $q_{\text {res }}$ shows that if $\Pi \hookrightarrow \mathscr{A}_{\text {res, },}(G)$ is a residual automorphic representation of $G(\mathbb{A})$ that is $\left(\mathfrak{m}_{G}, K\right)$-cohomological with respect to $\mathcal{M}$, then $q_{\min }<q_{\text {res }}$. Hence, Theorem 4.1 follows from our Theorem 3.2.

\section{Acknowledgements}

We would like to thank G. Harder for many inspiring discussions on this topic at the Erwin Schrödinger Institute in Vienna.

\section{References}

[Borel 1981] A. Borel, "Stable real cohomology of arithmetic groups II", pp. 21-55 in Manifolds and Lie groups (Notre Dame, In, 1980), edited by J. Hano et al., Progr. Math. 14, Birkhäuser, Boston, 1981. MR 83h:22023 Zbl 0483.57026

[Borel 2007] A. Borel, “Automorphic forms on reductive groups", pp. 5-40 in Automorphic forms and applications, edited by P. Sarnak and F. Shahidi, IAS/Park City Math. Ser. 12, Amer. Math. Soc., Providence, RI, 2007. MR 2008k:11045

[Borel and Jacquet 1979] A. Borel and H. Jacquet, "Automorphic forms and automorphic representations", pp. 189-202 in Automorphic forms, representations and L-functions (Corvallis, OR, 1977), edited by A. Borel and W. Casselman, Proc. Sympos. Pure Math. 33, Part 1, Amer. Math. Soc., Providence, RI, 1979. MR 81m:10055 Zbl 0414.22020 
[Borel and Wallach 1980] A. Borel and N. Wallach, Continuous cohomology, discrete subgroups, and representations of reductive groups, Annals of Mathematical Studies 94, Princeton University Press, New Jersey, 1980. MR 83c:22018 Zbl 0443.22010

[Borel et al. 1996] A. Borel, J.-P. Labesse, and J. Schwermer, "On the cuspidal cohomology of $S$-arithmetic subgroups of reductive groups over number fields", Compositio Math. 102:1 (1996), 1-40. MR 97j:11026 Zbl 0853.11044

[Enright 1979] T. J. Enright, "Relative Lie algebra cohomology and unitary representations of complex Lie groups”, Duke Math. J. 46:3 (1979), 513-525. MR 81i:22007 Zbl 0427.22010

[Franke 1998] J. Franke, "Harmonic analysis in weighted $L_{2}$-spaces", Ann. Sci. École Norm. Sup. (4) 31:2 (1998), 181-279. MR 2000f:11065 Zbl 0938.11026

[Franke and Schwermer 1998] J. Franke and J. Schwermer, "A decomposition of spaces of automorphic forms, and the Eisenstein cohomology of arithmetic groups", Math. Ann. 311:4 (1998), 765-790. MR 99k:11077 Zbl 0924.11042

[Grobner 2013] H. Grobner, "Residues of Eisenstein series and the automorphic cohomology of reductive groups", Compos. Math. 149:7 (2013), 1061-1090. MR 3078638

[Knapp and Vogan 1995] A. W. Knapp and D. A. Vogan, Jr., Cohomological induction and unitary representations, Princeton Mathematical Series 45, Princeton University Press, 1995. MR 96c:22023 Zbl 0863.22011

[Mœglin and Waldspurger 1989] C. Mœglin and J.-L. Waldspurger, "Le spectre résiduel de GL(n)", Ann. Sci. École Norm. Sup. (4) 22:4 (1989), 605-674. MR 91b:22028 Zbl 0696.10023

[Mœglin and Waldspurger 1995] C. Mœglin and J.-L. Waldspurger, Spectral decomposition and Eisenstein series, Cambridge Tracts in Mathematics 113, Cambridge University Press, Cambridge, 1995. MR 97d:11083 Zbl 0846.11032

[Rohlfs and Speh 2011] J. Rohlfs and B. Speh, "Pseudo Eisenstein forms and the cohomology of arithmetic groups III: residual cohomology classes", pp. 501-523 in On certain L-functions (Purdue University, IN, 2007), edited by J. Arthur et al., Clay Math. Proc. 13, Amer. Math. Soc., Providence, RI, 2011. MR 2012d:11123

[Vogan and Zuckerman 1984] D. A. Vogan, Jr. and G. J. Zuckerman, "Unitary representations with nonzero cohomology”, Compositio Math. 53:1 (1984), 51-90. MR 86k:22040 Zbl 0692.22008

Received December 20, 2013. Revised March 4, 2014.

HARALD GROBNER

FAKULTÄT FÜR MATHEMATIK

UNIVERSITÄT WIEN

OSKAR-MORGENSTERN-PLATZ 1

1090 WIEN

AUSTRIA

harald.grobner@univie.ac.at 


\title{
PACIFIC JOURNAL OF MATHEMATICS
}

\author{
msp.org/pjm
}

Founded in 1951 by E. F. Beckenbach (1906-1982) and F. Wolf (1904-1989)

\section{EDITORS}

V. S. Varadarajan (Managing Editor)

Department of Mathematics

University of California

Los Angeles, CA 90095-1555

pacific@math.ucla.edu

Paul Balmer

Department of Mathematics

University of California

Los Angeles, CA 90095-1555

balmer@math.ucla.edu

Daryl Cooper

Department of Mathematics

University of California

Santa Barbara, CA 93106-3080 cooper@math.ucsb.edu

Jiang-Hua $\mathrm{Lu}$

Department of Mathematics

Pokfulam Rd., Hong Kong jhlu@maths.hku.hk
The University of Hong Kong

Don Blasius

Department of Mathematics University of California

Los Angeles, CA 90095-1555

blasius@math.ucla.edu

Robert Finn

Department of Mathematics Stanford University

Stanford, CA 94305-2125

finn@math.stanford.edu

Sorin Popa

Department of Mathematics

University of California

Los Angeles, CA 90095-1555 popa@math.ucla.edu

Paul Yang

Department of Mathematics Princeton University

Princeton NJ 08544-1000

yang@math.princeton.edu

\section{PRODUCTION}

Silvio Levy, Scientific Editor, production@msp.org

\section{SUPPORTING INSTITUTIONS}

ACADEMIA SINICA, TAIPEI

CALIFORNIA INST. OF TECHNOLOGY

INST. DE MATEMÁTICA PURA E APLICADA

KEIO UNIVERSITY

MATH. SCIENCES RESEARCH INSTITUTE

NEW MEXICO STATE UNIV.

OREGON STATE UNIV.

\author{
STANFORD UNIVERSITY \\ UNIV. OF BRITISH COLUMBIA \\ UNIV. OF CALIFORNIA, BERKELEY \\ UNIV. OF CALIFORNIA, DAVIS \\ UNIV. OF CALIFORNIA, LOS ANGELES \\ UNIV. OF CALIFORNIA, RIVERSIDE \\ UNIV. OF CALIFORNIA, SAN DIEGO \\ UNIV. OF CALIF., SANTA BARBARA
}

\author{
Vyjayanthi Chari \\ Department of Mathematics \\ University of California \\ Riverside, CA 92521-0135 \\ chari@math.ucr.edu \\ Kefeng Liu \\ Department of Mathematics \\ University of California \\ Los Angeles, CA 90095-1555 \\ liu@math.ucla.edu \\ Jie Qing \\ Department of Mathematics \\ University of California \\ Santa Cruz, CA 95064 \\ qing@cats.ucsc.edu
}

These supporting institutions contribute to the cost of publication of this Journal, but they are not owners or publishers and have no responsibility for its contents or policies.

See inside back cover or msp.org/pjm for submission instructions.

The subscription price for 2014 is US \$410/year for the electronic version, and \$535/year for print and electronic.

Subscriptions, requests for back issues and changes of subscribers address should be sent to Pacific Journal of Mathematics, P.O. Box 4163, Berkeley, CA 94704-0163, U.S.A. The Pacific Journal of Mathematics is indexed by Mathematical Reviews, Zentralblatt MATH, PASCAL CNRS Index, Referativnyi Zhurnal, Current Mathematical Publications and Web of Knowledge (Science Citation Index).

The Pacific Journal of Mathematics (ISSN 0030-8730) at the University of California, c/o Department of Mathematics, 798 Evans Hall \#3840, Berkeley, CA 94720-3840, is published twelve times a year. Periodical rate postage paid at Berkeley, CA 94704, and additional mailing offices. POSTMASTER: send address changes to Pacific Journal of Mathematics, P.O. Box 4163, Berkeley, CA 94704-0163.

PJM peer review and production are managed by EditFLOW ${ }^{\circledR}$ from Mathematical Sciences Publishers.

\section{PUBLISHED BY}

mathematical sciences publishers

nonprofit scientific publishing

http://msp.org/

(C) 2014 Mathematical Sciences Publishers 


\section{PACIFIC JOURNAL OF MATHEMATICS}

Volume $268 \quad$ No. $1 \quad$ March 2014

AlEXANDRE PAIVA BARRETO

A transport inequality on the sphere obtained by mass transport

DARIO CORDERO-ERAUSQUIN

A cohomological injectivity result for the residual automorphic spectrum of $\mathrm{GL}_{n}$

HARALD GROBNER

Gradient estimates and entropy formulae of porous medium and fast diffusion equations for the Witten Laplacian

GUANGYUE HUANG and HAIZHONG LI

Controlled connectivity for semidirect products acting on locally finite trees

KeITH JoNES

An indispensable classification of monomial curves in $\mathbb{A}^{4}(\mathbb{k})$

ANARGYROS KATSABEKIS and IGNACIO OJEDA

Contracting an axially symmetric torus by its harmonic mean curvature

CHRISTOPHER KIM

Composition operators on strictly pseudoconvex domains with smooth symbol

HYUNGWOON KOO and SONG-YING LI

The Alexandrov problem in a quotient space of $\mathbb{U}^{2} \times \mathbb{R}$

AnA Menezes

Twisted quantum Drinfeld Hecke algebras

DEEPAK NAIDU

$L^{p}$ harmonic 1-forms and first eigenvalue of a stable minimal hypersurface

KEOMKYO SEO

Reconstruction from Koszul homology and applications to module and derived categories

RYO TAKAHASHI

A virtual Kawasaki-Riemann-Roch formula 